\title{
GOAT links dietary lipids with the endocrine control of energy balance
}

\author{
H. Kirchner ${ }^{1,2,{ }^{*},}$, J.A. Gutierrez ${ }^{3,}{ }^{*}$, P.J. Solenberg ${ }^{3,}{ }^{*}$, P.T. Pfluger ${ }^{1}$, T.A. Czyzyk ${ }^{3}$, J.A. \\ Willency $^{3}$, A. Schurmann ${ }^{2}$, H.G. Joost ${ }^{2}$, R. Jandacek ${ }^{1}$, J.E. Hale ${ }^{3}$, M.L. Heiman ${ }^{3}$, and M.H. \\ Tschöp ${ }^{1,2}$ \\ ${ }^{1}$ Depts. of Psychiatry and Medicine, Obesity Research Centre \& Genome Research Institute, \\ Univ. of Cincinnati College of Medicine, Cincinnati, OH, USA \\ 2 Dept. of Pharmacology, German Institute of Human Nutrition (DIfE), Nuthetal-Potsdam, \\ Germany
}

${ }^{3}$ Lilly Research Laboratories, Eli Lilly and Company, Indianapolis, IN, USA

\begin{abstract}
CNS nutrient sensing and afferent endocrine signalling are established as parallel systems communicating metabolic status and energy availability in vertebrates. The only afferent endocrine signal known to require modification with a fatty acid side chain is the orexigenic hormone ghrelin. We find that the ghrelin $O$-acyl transferase (GOAT) which is essential for ghrelin acylation, is regulated by nutrient availability, depends on specific dietary lipids as acylation substrates and modulates body fat mass in mice.
\end{abstract}

Two discoveries have softened the traditional differentiation between the classic model of nutrient sensing 1 and the concept of endocrine signals controlling energy status 2 and drawn attention to the regulation of energy homeostasis by circulating long chain fatty acids (LCFAs). Hotamisligil and colleagues recently reported that one specific adipocyte derived long chain fatty acid (C16:1n7), the lipokine palmitoleate, functions as a hormone regulating systemic insulin sensitivity 3 . A recent study followed with the discovery that a gastrointestinal lipid metabolite, $\mathrm{N}$-acylphosphatidylethanolamine (NAPE), can function as an endocrine signal which targets hypothalamic energy balance centers to control food intake, particularly when the acyl NAPE species is C16:0 4. Ten years after the discovery of the only orexigenic gut hormone ghrelin 5,6, this unique medium- chain fatty acid (MCFA)peptide chimera is now revealing itself as yet another nutrient-hormone hybrid with the specific role of linking macronutrient composition with CNS energy balance regulation. It is further intriguing that the only peptide hormone known to require a fatty acid modification 5 is also the only known afferent endocrine factor which depends on intra-neuronal fatty acid metabolism 7. Unique characteristics of the predominantly stomach derived ghrelin include

Users may view, print, copy, and download text and data-mine the content in such documents, for the purposes of academic research, subject always to the full Conditions of use:http://www.nature.com/authors/editorial_policies/license.html\#terms

Corresponding Author: Matthias H. Tschop, MD, Dept. of Psychiatry, Obesity Research Center \& Genome Research Institute,

University of Cincinnati, College of Medicine, Cincinnati, OH 45237.

* These authors contributed equally to this work 
appetite stimulation 6 , promotion of adiposity 6 , activation of hypothalamic NPY/AGRP neurons 8 , meal associated secretory peaks 9 , and its unique chemistry requiring acylation with MCFAs at its Ser 3 residue for activation of its only known receptor GHS-R1a 5.

We and others 10,11 recently identified the specific enzyme responsible for this important activation step and named it Ghrelin $O$-acyl Transferase (GOAT). Here we show that GOAT appears to function as a gastric lipid sensor linking selected ingested nutrients with hypothalamic energy balance regulation via the endocrine ghrelin system. Using murine genetic models with specific loss (GOAT-null) or gain (mice co-overexpressing human GOAT and human ghrelin) of GOAT function, we show that GOAT is required and sufficient to mediate the impact of certain dietary lipids on body adiposity. Using mass spectrometry and gene expression profiling, we also find that activation of the GOATghrelin system is triggered bya specific lipid-rich environment rather than by caloric deprivation. These observations suggest that the ghrelin/GOAT system informs the CNS about availability, rather than absence, of calories, thereby reversing the previously accepted model.

Since ghrelin plasma concentrations surge shortly before a meal, GOAT mRNA levels are expected to follow the ghrelin secretion pattern, increasing in the fasted state and decreasing postprandially. To test this hypothesis, four groups of C57BL/6 mice were fasted over a time course of 36 hours and gene expression patterns of both the GOAT gene (Mboat4) and the ghrelin gene $(\mathrm{ghrl})$ were examined. In addition, plasma concentrations of both acyl- and desacyl ghrelin were measured.

Surprisingly, gastric Mboat4 expression levels were highest under ad libitum (ad lib) conditions and decreased significantly with fasting for 12,24 or 36 hours $(\mathrm{p}=0.0205)$ (Fig. 1a). Ghrl mRNA also tended to be decreased after prolonged fasting and resembled the expression pattern of Mboat4, however, these changes did not reach statistical significance $(p=0.1875)$ (Fig. 1a). Notably, blood acyl ghrelin levels were not changed over the timecourse of fasting (Fig. 1b). However, blood concentrations of inactive des-acyl ghrelin increased significantly with fasting $(\mathrm{p}<0.0001)$ (Fig. 1b).

Due to the fact that there are 2 substrates for GOAT, ghrelin and MCFA, and that $g h r l$ expression is relatively constant during fasting, these data suggest that Mboat4 is downregulated in absence of available MCFA. To further test this hypothesis, we fed C57/BL/6 mice with a diet rich in glycerol triheptanoate, a medium chain triglyceride (MCT) containing heptanoic acid (C7:0), which does not occur endogenously. Nishi et al. showed previously that dietary lipids can directly influence ghrelin acylation 12. Similar to the results of Nishi et al. we show that heptanoic acid is used for ghrelin acylation. The C7ghrelin can be found in stomach tissue (Fig. 1c and d) and blood samples (Fig. 1e). Acylation of ghrelin with heptanoic acid appears to be at the expense of acylation with octanoic acid since octanoylated ghrelin was significantly reduced in the triheptanoate fed mice (blood $p=0.0126$; stomach $p=0.0006$ ) (Fig. 1d and e). The fact that heptanoylated ghrelin was more abundant than octanoylated ghrelin when fed this special diet supports our theory that dietary lipids play a major role in ghrelin acylation and that regulation of acyl ghrelin production and secretion is dependent on MCFA substrate. To investigate if small 
and acute changes of the amount of incoming dietary lipids have an impact on Mboat4 regulation or GOAT activity we then measured Mboat4 and ghrl expression as well as GOAT substrate (des-acyl ghrelin) and GOAT enzyme product (acyl-ghrelin) in wild type (WT) mice at several time points during the light cycle and the dark cycle (Supplementary Fig. 1a). Gene expression and GOAT enzyme activity tended to be more associated with the feeding period in the dark phase. Blood concentrations of acyl ghrelin were significantly increased after two hours of beginning of the dark phase ( $\mathrm{p}=0.0348)$ (Supplementary Fig. 1b) just after the most intense feeding period in mice during the first hours of the dark phase 13. To study the role of chronically increased food intake and obesity on the GOAT/ghrelin system we measured gastric gene expression of Mboat4 and $\mathrm{ghrl}$ in ob/ob mice fed ad lib and compared it to age-matched wild type mice. Neither Mboat 4 nor ghrl mRNA levels are changed by leptin deficiency-induced obesity (Supplementary Fig. 1c).

Next, we generated and examined GOAT knock out mice $\left(\right.$ Mboat $\left.^{-/-}\right)$(Supplementary Fig. 2) which lack acyl modified forms of ghrelin but have physiological levels of des-acyl ghrelin 10, to study the physiological role of GOAT on diets of various macronutrient compositions in comparison with wild type littermate mice. Mboat $^{-1-}$ mice fed standard chow diet had normal body weight (BW) and fat mass (FM) (Supplementary Fig. 3a). However, when fed high fat diet (HFD) for at least 8 weeks, the BW of Mboat $4^{-/-}$decreased significantly ( $51.55 \pm 0.69 \mathrm{~g} ; \mathrm{n}=8$ vs. $55.00 \pm 1.29 \mathrm{~g} ; \mathrm{n}=8 ; \mathrm{p}=0.0341)$ while no significant body composition changes were detected over that observation period and on that diet (Supplementary Fig. 3b). In an attempt to enhance and further clarify the phenotype, we therefore studied a new cohort of GOAT deficient mice and age matched littermate controls under modified dietary conditions. Since we and others 10,12 showed in previous experiments (see above) that MCT dietary lipids are a direct source for ghrelin acylation and since medium-chain fatty acids $(\mathrm{C} 8, \mathrm{C} 10)$ are substrates for GOAT-mediated ghrelin acylation 10 we wondered whether ghrelin activation can be enhanced by a diet rich in octanoic and decanoic acids. Therefore we designed a diet that contains $10 \%$ of digestible calories from glyceryl trioctanoate and glyceryl tridecanoate, hypothesizing that this diet would specifically trigger ghrelin octanoylation and decanoylation in the wild type mice. Mboat $4^{-/}$mice should not be influenced by this diet since these mice are not able to acylate ghrelin due to the global GOAT deletion. Feeding MCT diet to $\mathrm{Mboat}^{-1-}$ and wild type mice results in significant lower BW of Mboat $^{-{ }^{--}}$mice $(27.47 \pm 0.44 \mathrm{~g} ; \mathrm{n}=19$ vs. $29.39 \pm$ $0.64 \mathrm{~g} ; \mathrm{n}=15 ; \mathrm{p}=0.0161$ ) (Fig. 2a). Analysis of body composition revealed that the difference in $\mathrm{BW}$ can be explained by substantially lower FM $(17.90 \pm 1.74 \% ; \mathrm{n}=19$ vs. $24.14 \pm 2.45 \% ; n=15 ; p=0.0403$ ) (Fig. 2a). Such lower fat mass did not result from lower food intake which was actually higher in Mboat $^{-/-}$mice $(4.2 \pm 0.3 \mathrm{~g} / 24 \mathrm{~h}$ vs. $3.3 \pm 0.1 \mathrm{~g} /$ $24 \mathrm{~g} ; \mathrm{p}=0.0216$ ), but was likely based on higher energy expenditure (EE) in the light phase (light phase: $179.5 \pm 12.72 \mathrm{kcal} / 24 \mathrm{~h} / \mathrm{BW}^{0.75}$ vs. $157.3 \pm 14.74 \mathrm{kcal} / 24 \mathrm{~h} / \mathrm{BW}^{0.75} ; \mathrm{p}=0.032$ ) (Fig. 2a). Further, glucose homeostasis seemed yet unchanged in Mboat $4^{-/}$mice, since there were no significant differences in an intra peritoneal glucose tolerance test comparing WT and Mboat $^{-1}$-mice (Supplementary Fig. 3c).

In a third approach to uncover physiological GOAT function, we generated transgenic ( $\mathrm{Tg}$ ) mice designed to express the human ghrelin and human GOAT genes in the liver under 
control of the ApoE promoter. Multiple genetic lines were generated that stably carry and express both genes (data not shown). One line was selected for additional studies. These mice segregate both human ghrelin and GOAT genes in a Mendelian fashion, transmitting them stably to progeny, and have high circulating levels of 1-28 and 1-30 forms of des acyl and acetyl (C2) ghrelin. However, these mice lack octanoyl modified forms of human ghrelin in circulation (Supplementary Fig. 4a). We reasoned that dietary supplementation with MCT would allow for octanoylation of the hepatically expressed ghrelin forms, similar to our previous cell based studies 10. To determine if dietary MCT supplementation would lead to the generation of octanoylated human ghrelin forms, we exposed the $\mathrm{Tg}$ mice to a diet containing triglyceryl octanoate. When fed this diet, Tg mice displayed high circulating levels of 1-28 and 1-30 forms of des acyl, acetyl (C2), and octanoyl (C8) ghrelin (Supplementary Fig. 4b). Similarly, when the Tg mice were fed MCT diet, the mice produced large amounts of total ghrelin $(98.71+/-28.55 \mathrm{ng} / \mathrm{ml})$ compared to wild type controls $(0.61+/-0.33 \mathrm{ng} / \mathrm{ml}$ ) (Fig. 2c). Human octanoyl modified ghrelin levels were approximately $32 \mathrm{ng} / \mathrm{ml}$ and were undetectable in WT animals (Fig 2c). Such ghrelin overproduction led to significantly increased BW $(34.93 \pm 0.48 \mathrm{~g} ; \mathrm{n}=8$ vs. $31.88 \pm 1.32 \mathrm{~g}$; $\mathrm{n}=8 ; \mathrm{p}=0.0478)$ and $\mathrm{FM}(12.88 \pm 0.43 \mathrm{~g} ; \mathrm{n}=8$ vs. $10.29 \pm 0.84 \mathrm{~g} \mathrm{n}=8 ; \mathrm{p}=0.0158)$ in $\mathrm{Tg}$ mice in contrast to WT controls on the same diet over 4 weeks (Fig. 2b). Further, food intake did not differ between WT and Tg mice, but EE was significantly lower in Tg mice during both, the light and the dark phases $(\mathrm{p}=0.0068)$ (Fig. $2 \mathrm{~b})$. In addition, there was a strong trend towards increased respiratory quotient (RQ) during the light phase. Overall these data were indicating that $\mathrm{Tg}$ mice oxidize less fat than wild-type littermates (Fig. 2b). To demonstrate the dependence of ghrelin acylation on dietary MCT lipid availability we switched the Tg mice back to regular chow diet. After two weeks, the BW difference between $\mathrm{Tg}$ and wild type mice disappeared and no differences in EE and RQ were observed (Supplementary Fig. 5a). Therefore, our model of human ghrelin with human-GOAT overexpression and octanoylation is diet-inducible and reversible. To reassure the observed phenotype of the here described GOAT and ghrelin loss or gain of function models we generated additional populations of transgenic GOAT/ghrelin overexpressing and Mboat $4^{-/}$mice, further corroborating the body weight and fat mass phenotype differences in these larger groups of mutant and wild type mice (Supplementary Fig. 5b). To exclude the possibility that the here observed phenotype is due to a downstream effect of MCT feeding we compared body weight and composition of $\mathrm{Mboat}^{-/}$-mice on regular chow with age matched Mboat $4^{-/}$on MCT diet. Since Mboat $4^{-/}$mice are not able to acylate ghrelin any potential outcome of this comparison could be explained by a MCT diet effect. However, we found no phenotypic indication for non-GOAT specific effects of MCT diet that could have theoretically been mediated further downstream or through secondary effects of MCT independently from GOAT (Supplementary Fig 5c).

To identify mechanisms for the observed GOAT-mediated changes in energy expenditure and fat mass, we used the "switch on" transgenic GOAT/ghrelin mouse model where dietary exposure to MCT enriched diet is essential for the phenotype. Specifically, we compared genetic profiles relevant for fat oxidation as well as concentration of uncoupling protein-1 (UCP-1) and thyroxin. Our results point towards increased mitochondrial fat oxidation in skeletal muscle and potentially liver, but not brown adipose tissue (BAT), where neither 
gene expression nor protein concentrations of UCP-1 were changed (Supplementary Fig. 6a-d). Plasma concentration of thyroxin tended to be decreased in Tg mice, however the changes were not significant supporting a more muscle specific mechanism of decreased EE (Supplementary Fig. 6e).

We show here for the first time that GOAT mRNA levels decrease upon prolonged food deprivation and correct previous immunoassay-based models by proving with mass spectrometry-based analyses that in keeping with our GOAT expression studies, blood levels of acyl ghrelin are not increased during long term fasting. Mboat 4 mRNA levels are negatively correlated with des-acyl ghrelin concentrations in blood leading to the hypothesis that Mboat 4 expression or GOAT protein levels directly or indirectly repress translational control of $g h r l$.

Ghrl mRNA remains constant during the prolonged fasting but blood concentrations of desacyl ghrelin double. Since the latter appears to be a stable increase the data suggest translational control of the message with potential involvement of microRNA. The 2-fold decrease of Mboat4 mRNA after fasting does not result in lower acyl-ghrelin concentrations in blood. Therefore, GOAT activity might not be a bottleneck for ghrelin acylation in ad lib or fasting conditions. The measurement of GOAT activity and development of GOAT antibodies capable of determining levels of the mostly intra-membrane GOAT protein levels are necessary to confirm this theory.

Further, we show that dietary lipids are directly used for ghrelin acylation leading to unnatural heptanoyl ghrelin when feeding triheptanoate and more than double concentrations of octanoyl ghrelin when feeding a MCT diet. Mice lacking GOAT have decreased bodyweight and fat mass on MCT diet whereas transgenic ghrelin and GOAT overexpressors show increased body weight and fat mass, proving a role for the endogenous GOAT/ghrelin system in the control of energy balance and adiposity. We find that sufficient dietary supply of medium-chain triglycerides is important for ghrelin acylation since $\mathrm{Tg}$ mice are not able to produce large amounts of octanoylated human ghrelin when fed regular chow. Intriguingly, $\mathrm{Tg}$ mice on regular chow show substantial levels of inactive C2-acetyl modified ghrelin in the absence of octanoylated ghrelin suggesting that at least under these experimental conditions the GOAT fatty acid substrate for acylation, acetyl-CoA, is available for ghrelin acylation.

In humans, acylated ghrelin levels rise before a meal and drop afterwards, thus suggesting a role for ghrelin as hunger signal. A recent study however showed that plasma concentrations of acyl ghrelin do not increase under conditions of prolonged fasting 14. By establishing a new, superior sandwich ELISA with highly specific protection of acyl ghrelin against deacylation of the octanoyl side chain, they corroborated the well-known ghrelin secretion pattern with surges before a meal and suppression after the meal for the inactive and the active form of ghrelin. Nevertheless, during prolonged fasting for 36 hours or longer, plasma concentrations of acyl ghrelin were consistently at the basal level. Thorner's results therefore suggested that ghrelin may be regulated by separate secretion and activation processes and likely acts more as meal preparation cue rather than as a meal initiation factor or as a hunger signal that responds to persistent starvation. The interpretation of ghrelin 
being a meal preparation factor preparing the organism to optimally metabolize and store energy could possibly explain the reason why we do not find increased food intake in the GOAT/ghrelin transgenic mice. Further, gastric ghrelin might be more important for the regulation of energy metabolism in muscle and liver.

Prolonged food deprivation in our studies also did not lead to increased acyl ghrelin blood levels. In addition, neither gastric Mboat4 expression nor $\mathrm{ghrl}$ mRNA levels were upregulated during fasting as would have been expected based on the traditional model of ghrelin function. Taken all together our results therefore do not point towards a prominent role for both $\mathrm{ghrl}$ and Mboat $4 \mathrm{mRNA}$ expression as a key process for the generation of a hunger signal that indicates an empty stomach. Ghrelin acylation and thereby activation however appear to be influenced substantially by food intake and fatty acid composition of the ingested food. It remains unclear why endogenously derived fatty acids mobilized from adipose stores are apparently not used to massively acylate ghrelin during fasting. MCTs, the substrate for GOAT 10, can principally be produced during metabolism of long chain fatty acids by beta oxidation where the long chain fatty acid is shortened by 2 carbons with each cycle in an impressively rapid process 15 . Only recently, more data on modulation of fatty acid profiles have become available, but they usually were measured after a substantial fast rather than between meals when acyl ghrelin levels would be impacted in a meaningful manner 14. However, even after 12 hours of fasting MCFAs remain present in plasma 15 and are not completely oxidized. We therefore conclude, based on the existing body of published data in combination with our results, that ghrelin acylation and the secretion of acylated ghrelin likely represent two independent processes and that GOAT/ghrelin might act as a lipid sensor that is activated when certain fatty food is consumed.

Intriguingly, ghrelin secreting cells in the gastrointestinal tract are of the closed-and opentype with the open-type cells more abundant in the duodenum where fatty acids are absorbed. Thus, it is possible that dietary lipids are taken up by the open-type ghrelin cells and directly used for ghrelin acylation. Follow up studies providing improved insight into such mechanistic details could help to understand and more efficiently explore the rapid metabolic benefits of some bariatric surgeries which are becoming increasingly popular for the treatment of severe obesity with type 2 diabetes mellitus.

Two recently published studies also highlight the emerging importance of dietary fat as a substrate for endogenous modulators of energy balance: The lipid mediator Oleoylethanolamide (OEA) and its precursor N-acylphosphatidylethanolamine (NAPE) both affect food intake and energy metabolism utilizing dietary fat as a substrate and intriguingly, an acyltransferase is involved in OEA activation 4,16. Medium-chain triglycerides exhibit a number of particular features: a) they are hydrolyzed much more rapidly than long-chain triglycerides in the intestinal lumen. b) The presence of pancreatic enzymes and bile salts are not required for absorption of MCT; and c) MCTs are transported via the portal vein as free fatty acids (FFA) bound to albumin whereas LCTs must undergo esterification and chylomicron formation and are transported in the lymph. In nature, MCTs are found in milk along with LCT and because MCTs are more efficiently digested, some infant formulae are enriched with MCT. For the same reason MCT therapy is indicated for patients with malabsorption 17. Our findings indicate that the orexigenic, positive energy balance- 
promoting GOAT/ghrelin system is triggered by dietary MCTs, and offer a new perspective for the potential role of MCTs in milk and infant formula that deserves further study.

A naturally high source of MCT is coconut. Studies conducted in the Polynesian Islands where meals are composed of up to $60 \%$ from coconut show high prevalence of obesity and dyslipidemia which can be linked to the high intake of coconut products. Although ghrelin was not measured in this population it can be assumed that the extreme MCT intake triggers ghrelin acylation possibly leading to increased peripheral lipid storage and hypercholesterolemia 18, two phenomena that are associated with ghrelin signaling 6,19.

The new model we are proposing with GOAT/ghrelin as a lipid sensor appears logical when reviewing the main functions of ghrelin; ghrelin triggers growth hormone release and lipogenesis, two features that seem to be irrational during hunger. It would make more sense for an organism to allow for growth and support fat storage while nutrients are coming in. Therefore, and while more studies are needed to completely dissect acyl- from des-acyl ghrelin functions or the ghrelin activation from ghrelin secretion processes, we propose that ghrelin's predominant physiological function may not necessarily, at least not exclusively, be that of a hunger signal that reflects an empty stomach as previously assumed. Based on the data presented here, it seems more likely that the GOAT-ghrelin system acts as a nutrient sensor by using readily absorbable medium-chain fatty acids to signal to the brain that high caloric food is available, leading to optimization of nutrient partitioning and growth signals (Supplementary Fig. 7).

Our findings highlight the importance of identifying the physiological role of "inactive" desacyl ghrelin. The fact that des-acyl ghrelin appears to be secreted in highly regulated fashion in response to caloric deprivation (pls. also see Fig. $1 \mathrm{~b}$ and reference 14) suggests the likely existence of a yet to be discovered physiological function, which based on indirect evidence could be related to glucose metabolism 20 and might involve an additional ghrelin receptor 19.

Finally, the gastrically expressed GOAT offers an accessible and elegant drug target for the treatment of metabolic diseases: Since loss and gain of function models prove its role in diet-induced adiposity regulation, GOAT modulators may offer potential as novel antiobesity drugs or anti-cachexia therapeutics.

All studies were approved by and performed according to the guidelines of the Institutional Animal Care and Use Committee (IACUC) of the University of Cincinnati and the Eli Lilly Company, Indianapolis.

\section{Supplementary Material}

Refer to Web version on PubMed Central for supplementary material.

\section{Acknowledgments}

The authors would like to thank James E. Baker and Jenna Holland for their outstanding technical support. This manuscript was supported by the Leibniz Graduate College (HK) and by National Institutes of Health/National Institute of Diabetes and Digestive and Kidney Diseases Grant R01-DK069987 (to MHT). 


\section{References}

1. Obici S, et al. Central melanocortin receptors regulate insulin action. J Clin Invest. 2001; 108:107985. [PubMed: 11581309]

2. Schwartz MW, Woods SC, Porte D Jr, Seeley RJ, Baskin DG. Central nervous system control of food intake. Nature. 2000; 404:661-71. [PubMed: 10766253]

3. Cao $\mathrm{H}$, et al. Identification of a lipokine, a lipid hormone linking adipose tissue to systemic metabolism. Cell. 2008; 134:933-44. [PubMed: 18805087]

4. Gillum MP, et al. N-acylphosphatidylethanolamine, a gut- derived circulating factor induced by fat ingestion, inhibits food intake. Cell. 2008; 135:813-24. [PubMed: 19041747]

5. Kojima M, et al. Ghrelin is a growth-hormone-releasing acylated peptide from stomach. Nature. 1999; 402:656-60. [PubMed: 10604470]

6. Tschop M, Smiley DL, Heiman ML. Ghrelin induces adiposity in rodents. Nature. 2000; 407:90813. [PubMed: 11057670]

7. Lopez M, et al. Hypothalamic fatty acid metabolism mediates the orexigenic action of ghrelin. Cell Metab. 2008; 7:389-99. [PubMed: 18460330]

8. Kamegai J, et al. Chronic central infusion of ghrelin increases hypothalamic neuropeptide $\mathrm{Y}$ and Agouti-related protein mRNA levels and body weight in rats. Diabetes. 2001; 50:2438-43. [PubMed: 11679419]

9. Tschop M, et al. Post-prandial decrease of circulating human ghrelin levels. J Endocrinol Invest. 2001; 24:RC19-21. [PubMed: 11434675]

10. Gutierrez JA, et al. Ghrelin octanoylation mediated by an orphan lipid transferase. Proc Natl Acad Sci U S A. 2008; 105:6320-5. [PubMed: 18443287]

11. Yang J, Brown MS, Liang G, Grishin NV, Goldstein JL. Identification of the acyltransferase that octanoylates ghrelin, an appetite-stimulating peptide hormone. Cell. 2008; 132:387-96. [PubMed: 18267071]

12. Nishi $Y$, et al. Ingested medium-chain fatty acids are directly utilized for the acyl modification of ghrelin. Endocrinology. 2005; 146:2255-64. [PubMed: 15677766]

13. Drazen DL, Vahl TP, D'Alessio DA, Seeley RJ, Woods SC. Effects of a fixed meal pattern on ghrelin secretion: evidence for a learned response independent of nutrient status. Endocrinology. 2006; 147:23-30. [PubMed: 16179409]

14. Liu J, et al. Novel ghrelin assays provide evidence for independent regulation of ghrelin acylation and secretion in healthy young men. J Clin Endocrinol Metab. 2008; 93:1980-7. [PubMed: 18349056]

15. Dole VP, Rizack MA. On the turnover of long-chain fatty acids in plasma. J Lipid Res. 1961; 2:90-91.

16. Fu J, Oveisi F, Gaetani S, Lin E, Piomelli D. Oleoylethanolamide, an endogenous PPAR-alpha agonist, lowers body weight and hyperlipidemia in obese rats. Neuropharmacology. 2005; 48:1147-53. [PubMed: 15910890]

17. Zurier RB, Campbell RG, Hashim SA, Van Itallie TB. Use of medium-chain triglyceride in management of patients with massive resection of the small intestine. N Engl J Med. 1966; 274:490-3. [PubMed: 5904288]

18. Prior IA, Davidson F, Salmond CE, Czochanska Z. Cholesterol, coconuts, and diet on Polynesian atolls: a natural experiment: the Pukapuka and Tokelau island studies. Am J Clin Nutr. 1981; 34:1552-61. [PubMed: 7270479]

19. Pfluger PT, et al. Simultaneous deletion of ghrelin and its receptor increases motor activity and energy expenditure. Am J Physiol Gastrointest Liver Physiol. 2008; 294:G610-8. [PubMed: 18048479]

20. Gauna C, et al. Unacylated ghrelin acts as a potent insulin secretagogue in glucose-stimulated conditions. Am J Physiol Endocrinol Metab. 2007; 293:E697-704. [PubMed: 17578884] 

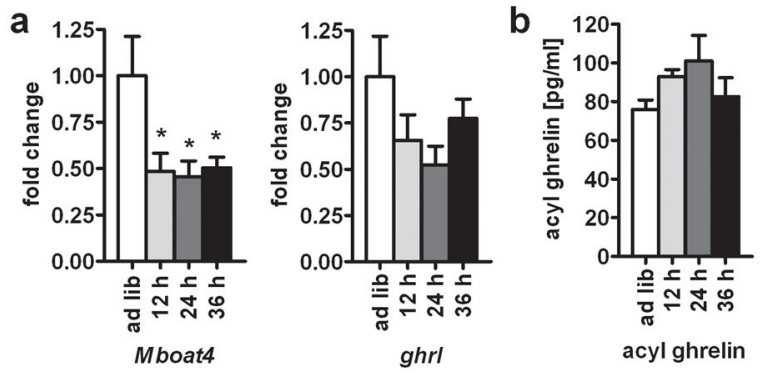

c
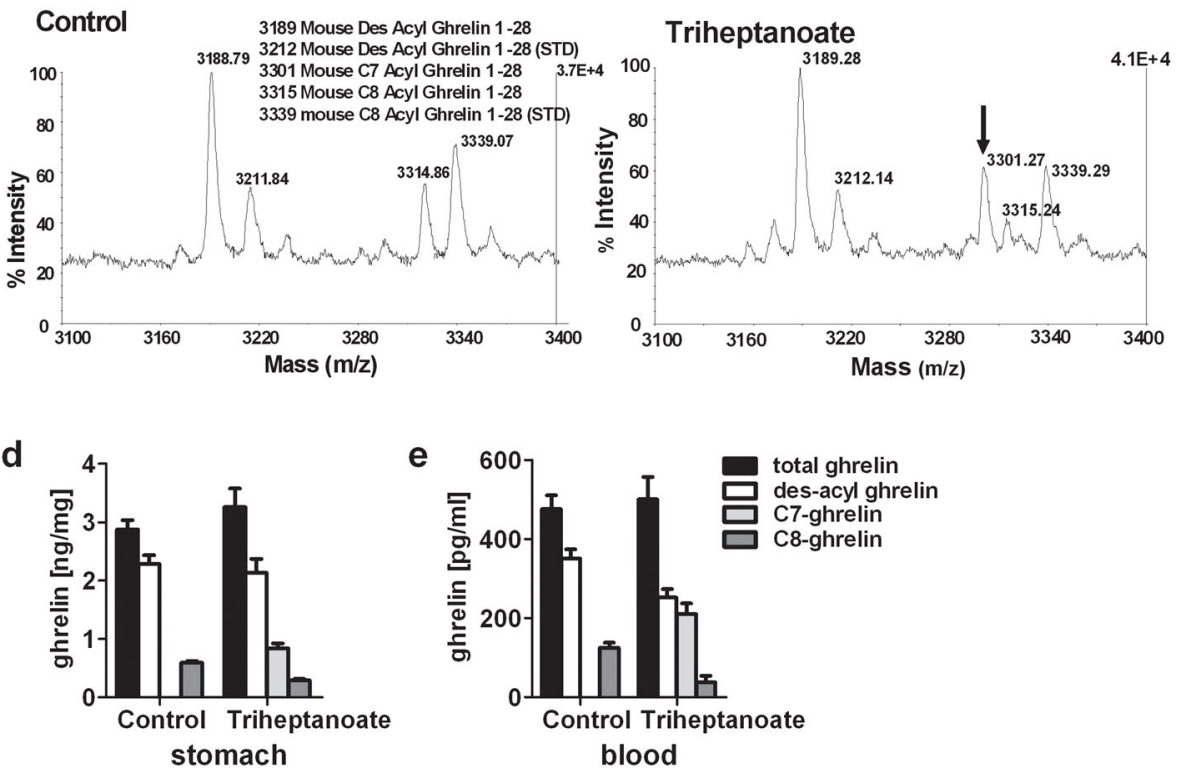

Figure 1.

Long-term fasting decreases Mboat4 expression and does not increase ghrelin acylation. (a) Fold change in gastric Mboat 4 and $\mathrm{ghrl}$ mRNA levels of C57BL-6 mice measured with pPCR after fasting for $12(n=6), 24(n=5)$, and $36(n=8)$ hours compared to mice fed chow ad lib (n=7). (b) Blood concentrations of acyl and des-acyl ghrelin in the same mice after fasting for $12 \mathrm{~h}, 24 \mathrm{~h}$, and $36 \mathrm{~h}$ or feeding ad lib. (c) Ghrelin immunoprecipitation MALDIToF MS (IPMS) analyses of stomachs from mice exposed to normal (control) or glyceryl triheptanoate (C7)- containing diets. Downward arrow denotes peak corresponding to heptanoyl-modified ghrelin peptide (m/z 3301). Peaks at m/z 3189, 3212, 3315, and 3339 correspond to mouse forms of des-acyl ghrelin, des acyl ghrelin standard, octanoylated ghrelin and octanoylated ghrelin standard, respectively. (d) Total concentration of ghrelin forms isolated form stomachs of C57BL-6 mice fed chow or triheptanoate enriched diet. (e) Total blood concentration of ghrelin forms in C57BL-6 mice fed chow or glyceryl triheptanoate-enriched diet. All data are represented as mean \pm SEM. 
a
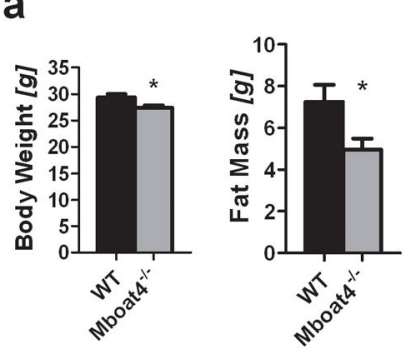

b
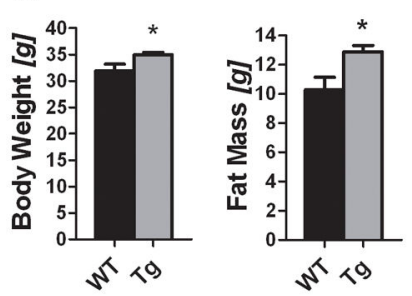
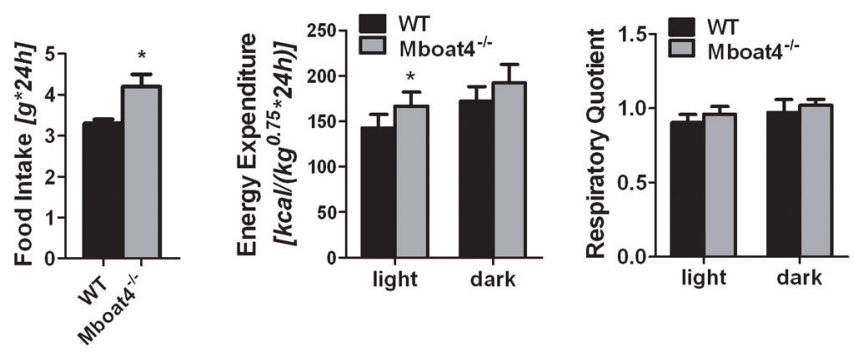

C
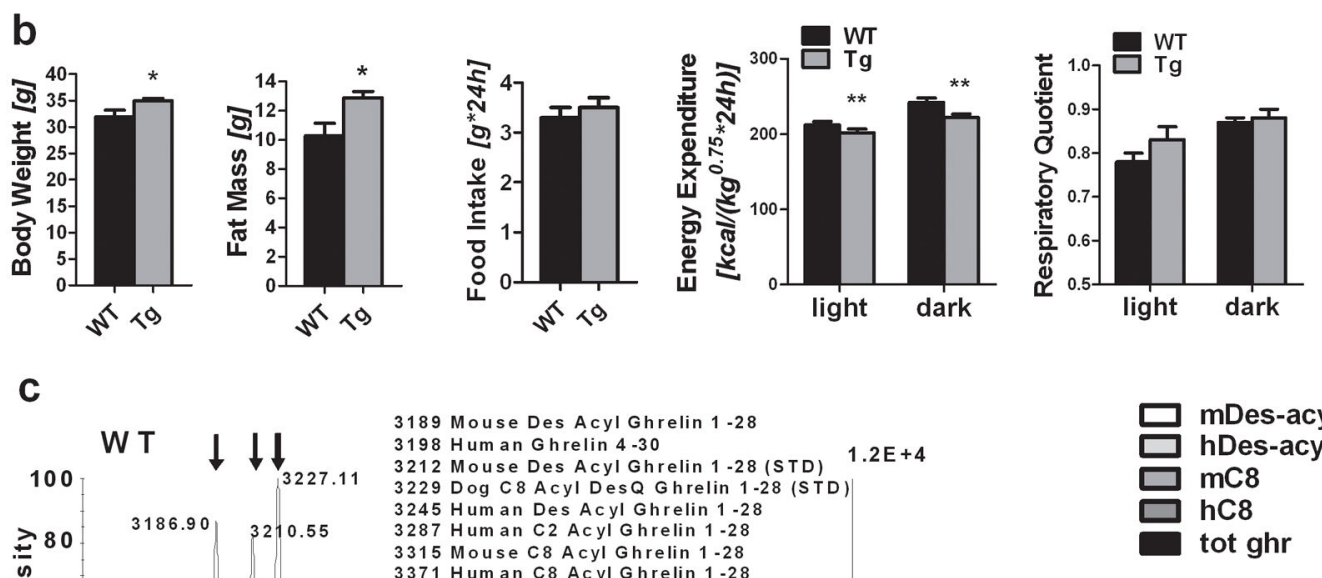

Figure 2.

GOAT regulates energy homeostasis (a) BW, FM (measured with NMR), FI, EE and RQ of WT and Mboat $4^{-1-}$ mice chronically fed MCT diet. (b) BW, FM (measured with NMR), FI, EE and RQ of WT and GOAT/ghrelin Tg mice chronically fed MCT diet. (c) Ghrelin IPMS analyses of blood from either WT or GOAT/ghrelin Tg mice exposed long term to MCT diet. Downward arrows denote endogenous mouse des acyl (m/z 3189) or octanoyl modified $(\mathrm{m} / \mathrm{z} 3315)$ ghrelin forms or the standard peptides $(\mathrm{m} / \mathrm{z} 3212,3229)$ used in these studies. Peaks at $\mathrm{m} / \mathrm{z} 3245,3287$, and 3371 represent human des acyl-, acetyl-, and octanoylmodified forms of ghrelin 1-28. Peaks at $\mathrm{m} / \mathrm{z} 3429,3471$, and 3555 correspond to human 
des acyl-, acetyl-, and octanoyl-modified forms of ghrelin 1-30. Bar graphs represent mean ghrelin concentrations of WT $(n=8)$ and $\mathrm{Tg}(\mathrm{n}=8)$ mice fed MCT diet. All data are represented as mean \pm SEM. 